\title{
Influence of the filler content on the free nanohole volume in epoxy-based composites
}

\author{
S. Tognana ${ }^{*}$, W. Salgueiro ${ }^{1}$, A. Somoza ${ }^{1}$, A. J. Marzocca ${ }^{2}$ \\ ${ }^{1}$ IFIMAT - Universidad Nacional del Centro de la Provincia de Buenos Aires, Pinto 399, (B7000GHG) Tandil, Argentina \\ and Comisión de Investigaciones Científicas de la Provincia de Buenos Aires, calle 526 entre 10 y 11, La Plata, \\ Argentina \\ ${ }^{2}$ LPMP y MC, Departamento de Física, Facultad de Ciencias Exactas y Naturales, Universidad Nacional de Buenos Aires, \\ Ciudad Universitaria, Pabellón I, C1428EGA Buenos Aires, Argentina
}

Received 29 June 2012; accepted in revised form 11 September 2012

\begin{abstract}
A study on free nanohole volumes in particulate epoxy matrix composites as a function of the aluminum particles content is presented. Specifically, the influence of the filler content in the epoxy matrix on the nanohole volume is analyzed in terms of the mechanical and morphological properties of the composites fabricated. Nanoholes data were measured using positron annihilation lifetime spectroscopy recently published by the authors. Applying the Park-Earmme micromechanical model, these data are interpreted in terms of the thermal stresses generated during the curing process applied during fabrication. Some input parameters of the model were experimentally obtained. In order to obtain a satisfactory description of the evolution of the free nanohole volume in the whole range of filler contents, a contribution due to the matrix-particle interphases is taken into account in the micromechanical model. To this aim, specific information on the interphases was obtained using atomic force microscopy (AFM), scanning electron microscopy (SEM), differencital scanning calorimetry (DSC) and a free-constraint analysis of the positron lifetime data.
\end{abstract}

Keywords: polymer composites, epoxy, free volume, positron annihilation

\section{Introduction}

The use of polymer matrix composites in different technological applications is of interest due to their very good mechanical properties, long life in service and optimization of cost. The different applications of a composite determin the different kinds and geometries of the inclusions (i.e., fillers) as well as of the polymeric matrix. Specifically, many epoxy matrix composites filled with inorganic particles composites have been developed. These systems have been extensively studied in order to understand the influence of inorganic particles added to the matrix on properties of these composites (mechanical, thermal, optical, etc.) [1].
Epoxy matrix composites are fabricated performing a cure treatment at high temperature of the resin with the addition of a specific hardener and particles used as filler. This process mainly defines the network structure and, consequently, the final properties of a composite [2]. As an example, strong residual stresses can be generated during curing as a consequence of the thermal and mechanical mismatches between the constituent phases of the composite.

The residual stresses have been studied for some decades, using experimental and theoretical tools. In this last case, different theories and calculation methods have been developed. In particular, Eshelby [3] proposed a method to calculate the stress field

\footnotetext{
${ }^{*}$ Corresponding author, e-mail: stognana@exa.unicen.edu.ar (C) BME-PT
} 
around an anisotropic particle embedded in a matrix of a general composite. Selsing reported [4] a simply equation for the residual stresses around a single inclusion in ceramic-based composites. Besides, a generalized self-consistent method was proposed and extensively used for the investigation of the physical properties of two-phase composites [5]. In particular, Park and Earmme [6] used some of these methods to investigate the residual stresses produced by a temperature variation during the manufacturing process of metal-matrix composites. These methods have also been applied to polymermatrix composites $[7,8]$.

On the other hand, besides the residual stresses, the inclusion of particles in a polymeric matrix can generate other structural changes in the material. In composites, the filler-matrix interphases (i.e. a region around the surface between the constituent phases of the composites) have different characteristics with respect to the matrix. In fact, around the inclusion surface there is a region where the local properties are different of the bulk matrix properties [9]. In the interphase region from the matrix to the filler such differences become gradual. In polymers, these interphases can be formed during the curing process. However, the nature of the interphase remains as a field of research. The existence of this so-called third phase can influence in an important way the total properties of the composite. This concept was particularly developed in a three phase model that allowed the study of the mechanical properties in polymer matrix-metal particles composites [10]. In epoxy matrix composites, residual stresses and the interphase formation have experimentally been studied using different techniques [11]. However, it is difficult to find a proper technique that allows the study of both processes at the same time.

Some of the authors of the present work have studied the influence of the residual stresses on the free volume in polymer-matrix composites, taking advantage of the high specificity of Positron Annihilation Lifetime Spectroscopy (PALS) $[8,12,13]$. PALS is practically the only experimental technique that allows to directly measure the size of nanohole forming the free volume [14].

According to the common interpretation of PALS spectra in polymers, the nanoholes sizes $V_{\mathrm{H}}$ can be directly estimated using a well-recognized semiempirical equation through the long-lived lifetime component, attributed to ortho-Ps (ortho-Positron- ium) annihilation by pick-off trapped in the free nanohole volumes $[14,15]$. This lifetime $\tau_{\mathrm{o}-\mathrm{Ps}}$ component is usually used as the experimental input and by means of a simple quantum mechanical model the radius of the nanohole can be obtained from Equation (1) $[14,15]$ :

$$
\tau_{\mathrm{o}-\mathrm{Ps}}=0.5\left[1-\frac{R}{R+\delta R}+\frac{1}{2 \pi} \operatorname{sen}\left(\frac{2 \pi R}{R+\delta R}\right)\right]^{-1}
$$

in which $\delta R$ is the thickness $(0.166 \mathrm{~nm})$ of the electron layer that constitutes the wall of the nanohole and can overlap with the o-Ps wave function. The nanohole volume can be computed as $V_{\mathrm{H}}=4 \pi R^{3} / 3$. It should be emphasized that the free volume is a concept used to explain the variation of several properties of a polymer under changes of temperature or pressure [16]. Also, it has been related to the structure and reticulation of the polymeric chains $[17,18]$. However, the results obtained in those materials cannot be directly linked with the phenomena studied in the present work.

In this work, we go deeper into the analysis of experimental PALS results we reported in [13] on the variation of the free nanohole volume in particulate epoxy matrix as a function of the aluminum particles content. As a result, it was found that the free volume depends on the thermal residual stresses and the interphase formation in the fabricated composites containing different filler contents (from the blank epoxy resin to $30 \%$ ). For the analysis of the experimental data, it was necessary to utilize different well-known micromechanical models as well as specific experimental techniques. The procedure we followed in approaching the objective of the present work was developed in different steps. Firstly, a micromechanical model, based on different approximations reported in the literature, allowed us to obtain the residual stresses generated into the composite matrix as a consequence of the addition of inclusions. In order to calculate the stresses values as a function of the filler content, previously reported values of the Young modulus, obtained by authors of the present work using the impulse excitation technique (IET), were used as input parameters into the micromechanical model. However, when the inclusion volumetric fraction was higher than $15 \%$ this model failed in describing the behavior of the experimental free nanohole volume data. Therefore, in a second step the influence of the inclusion-matrix interphases on the free nanohole 
volume in the different composites fabricated was added to the model mentioned taking into account two contributions to the free nanohole volume; i.e. matrix and interphases. To this aim, experimental information regarding interphases was obtained using atomic force microscopy (AFM) and differential scanning calorimetry (DSC). These results were then used into a reformulated micromechanical model allowing a satisfactory description of the free nanohole volume in the whole range of the filler content. It is important to emphasize that a quantitative analysis on the contribution of the inclusion-epoxy matrix interphases to the free nanohole volumes is one of the most important contributions of this work to the literature, as well as a comprehensive discussion on the variation of the nanohole volume when different amounts of inorganic filler are added to the epoxy matrix of the composite.

\section{Experimental}

\subsection{Sample preparation}

The composites studied were prepared using as matrix diglycidyl ether of bisphenol A (DGEBA) epoxy resin, cured with an anhydride (MTHPA) and an accelerator (tertiary amine). DGEBA (100 pbw) and MTHPA (90 pbw) were mixed and stirred at room temperature in vacuum during 30 minutes. Then, the accelerator $(0.7 \mathrm{pbw})$ was added and the mixture was stirred again during 5 minutes under vacuum. Finally, a required amount of aluminum particles was added and the mixture was stirred under vacuum until to reach a good dispersion of the particles in the resin. The compound was poured in a Pyrex tubular mold and kept in horizontal position in a tubular oven.

With the help of a device especially developed a rotation was imposed to the molds in order to avoid the settling down of the aluminum particles during the curing. In the curing process, the mixture was heated up to $393 \mathrm{~K}$ with a $0.8 \mathrm{~K} / \mathrm{min}$ rate, and at the end of the heating process, the samples were kept at a constant temperature ( $393 \mathrm{~K}$ ) for $14 \mathrm{~h}$. The composites were prepared using aluminum powder with particle diameters between 106 and $125 \mu \mathrm{m}$ and with a filler volume fraction, $\phi$, ranging from 0 to $30 \%$ in $5 \%$ steps. More details about the preparation process are given in $[8,12]$.

For microscopy measurements, the samples were cut using a low speed diamond saw and then pol- ished in several steps, starting with 1200 grit grinding paper followed by 3 and $1 \mu \mathrm{m}$ diamond particles and 0.3 and $0.05 \mu \mathrm{m}$ alumina particles/water suspensions.

\subsection{Atomic force and scanning electron microscopies}

SEM images were taken with a FE-SEM, Zeiss DSM 982 Gemini microscope.

AFM tests were carried out in tapping mode in a Digital Instruments Nanoscope III. The tests were performed using a single beam cantilever provided by an etched silicon nitride probe, in a nitrogen atmosphere. During the scanning, the topography and phase shifts, i.e., changes in the phase angle of vibration respect to the phase angle of the freely oscillating cantilever, were simultaneously recorded. The phase image allows revealing differences in surface properties of the material which in nature are currently qualitative. The AFM data were analyzed by using the WSxM 3.0 software [19].

\section{Results and discussion}

\subsection{PALS}

In Figure 1, the variation of the average free nanohole volume as a function of the filler volume fraction is shown. Though these data were already published in a previous work [13], in order to facilitate the reading and put major emphasis in the key issues of the present work, we decided to present them and to give a brief analysis of the experimental data again. As can be seen, there is a complex and non-monotonic behavior of $V_{\mathrm{H}}$ when $\phi$ increases. To analyze it, the plot was divided into three regions: in the first, when the filler content is lower than

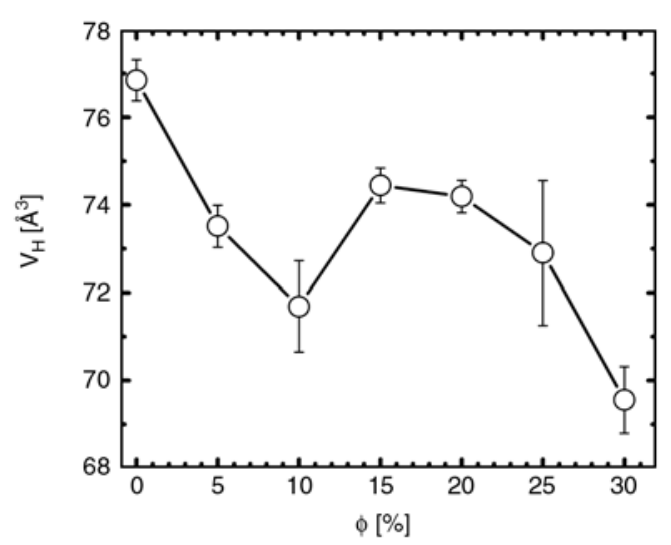

Figure 1. Average free nanohole volume as a function of the filler content measured, using positron annihilation lifetime spectroscopy (after [13]) 
$10 \%, V_{\mathrm{H}}$ rapidly decreases when the filler content increases; in the second region, an increment of $V_{\mathrm{H}}$ until $\phi$ reaches a value around $15 \%$; and for higher volume fractions (third region) the average free nanohole volume decreases, initially in a slight way and then in a more noticeably mode.

A similar behavior has been previously reported and discussed by some authors of the present work. In that paper, Goyanes et al. [8] discussed the results obtained in terms of the average thermal stresses occurring in the matrix as a consequence of the thermal mismatch due to the presence of filler particles. However, the authors could only explain the $V_{\mathrm{H}}$ decreasing behavior for the first region (i.e., till $\phi$ values up to $10-15 \%$ ) of the curve above described. To go further into the analysis of the data presented in Figure 1 and taking as a starting point the previous work of Goyanes et al. [8] and Tognana et al. [13], it was necessary to obtain additional experimental evidences. Besides, a deeper analysis of the data using well-known micromechanical models was carried out. Specifically, experimental information obtained using calorimetric and mechanical properties measurements was used as input parameters in such models.

In the following sections, different contributions to solve the same original problem, specifically to explain the behavior of the free nanohole volume against the filler content in the whole volume fraction range, are given.

\subsection{Thermal residual stresses around a particle}

It is well-known that during the fabrication process of the composites, strong residual stresses are produced when cooling the material from the cure temperature to that of service. These residual stresses are caused by the difference between the coefficients of thermal expansion (CTE) of the matrix and the inclusion as well.

Selsing [4] calculated the residual stresses, induced by a change in the temperature $(\Delta T)$, produced around a single spherical inclusion of radius $r_{\mathrm{f}}$ imbedded within an infinite isotropic elastic matrix. In order to analyze the stresses above mentioned, the radial stress in the matrix is can be calculated by Equation (2):

$\sigma_{\mathrm{rr}}^{\mathrm{M}}=-P \cdot\left(\frac{r_{\mathrm{f}}}{r}\right)^{3}$ where $P$ is the hydrostatic pressure in the inclusion and $r$ is the distance since the center of the inclusion. The value of $P$ can be obtained using the following Equation (3) [4]:

$$
P=\frac{\left(C T E_{\mathrm{M}}-C T E_{\mathrm{F}}\right) \Delta T}{\frac{1+v_{\mathrm{M}}}{2 E_{\mathrm{M}}}+\frac{1-2 v_{\mathrm{F}}}{E_{\mathrm{F}}}}
$$

where CTE is the coefficient of thermal expansion, and the subscripts $M$ and $F$ refer to the matrix and the particle, respectively. In the inclusion, the radial stress is independent of the $r$ parameter (see Equation (4)), so Selsing [4] concluded that:

$\sigma_{\mathrm{rr}}^{\mathrm{F}}=-P$

On the other hand, the tangential component of the residual stresses in the matrix as well as in the inclusion can be calculated as shown by Equations (5) and (6) (for details see [4]):

$$
\begin{aligned}
& \sigma_{\theta \theta}^{\mathrm{M}}=\sigma_{\phi \phi}^{\mathrm{M}}=\frac{P}{2}\left(\frac{r_{\mathrm{f}}}{r}\right)^{3} \\
& \sigma_{\theta \theta}^{\mathrm{F}}=\sigma_{\phi \phi}^{\mathrm{F}}=-P
\end{aligned}
$$

In a composite containing a high amount of particles in the matrix, if the stress fields around each particle do not overlap each other both components of the residual stresses are used. In such a sense, from a study on ceramic matrix composites [20] it was reported that the above expressions for the residual stresses were valid for composites containing up to approximately $15 \%$ of filler volume fraction. Taking into account that result, we could conclude that the use of a simple model like that proposed by Selsing [4] is not enough to obtain reliable information on the evolution of the residual stresses in our composites. The filler content is higher than a limit given by the overlapping of the stress fields of the individual particles we have found to be around $15 \%$. For this reason, from the micromechanical point of view we have used other models which allowed us to obtain a satisfactory description of the realistic problem we were dealing with. In the next two Sections they are discussed in detail.

\subsection{Average thermal stresses}

To go further into the analysis of the thermal stresses present in a composite, in this Section we will dis- 
cuss them in terms of the existence of a higher amount of inclusions into the materials.

Park and Earmme [6] modeled the thermal residual stresses using the generalized self-consistent method proposed by Christensen and Lo [5] to study other properties of a general kind of particulate materials. Basically, a spherical inclusion is considered to be perfectly bounded to a spherical shell which is embedded in an effective medium. The radius of the inclusion and the shell are $r_{\mathrm{f}}$ and $r_{\mathrm{b}}$, respectively. Then, $\left(r_{\mathrm{f}} / r_{\mathrm{b}}\right)^{3}$ represents the filler content, $\phi$. In the model, it is assumed that the mechanical and thermal properties of the effective medium are inbetween that corresponding to the matrix and that of the inclusion. As a result, Park and Earmme estimated the stresses in the three regions (i.e., matrix, particles and effective medium). In particular, they reported that the stress into the matrix is given by Equations (7) and (8):

$\sigma_{\mathrm{rr}}^{\mathrm{M}}=\frac{12 K_{\mathrm{F}} \varepsilon_{\mathrm{ij}}^{\mathrm{F}}}{D}\left(I-I I\left(\frac{r_{\mathrm{f}}}{r}\right)^{3}\right)+\frac{12 K_{\mathrm{M}} \varepsilon_{\mathrm{ij}}^{\mathrm{M}}}{D}\left(I I I+I V\left(\frac{r_{\mathrm{f}}}{r}\right)^{3}\right)$

$$
\begin{gathered}
\sigma_{\theta \theta}^{\mathrm{M}}=\sigma_{\phi \phi}^{\mathrm{M}}=\frac{12 K_{\mathrm{F}} \varepsilon_{\mathrm{ij}}^{\mathrm{F}}}{D}\left(I+I I\left(\frac{r_{\mathrm{f}}}{r}\right)^{3}\right)+ \\
+\frac{12 K_{\mathrm{M}} \varepsilon_{\mathrm{ij}}^{\mathrm{M}}}{D}\left(I I I-I V\left(\frac{r_{\mathrm{f}}}{r}\right)^{3}\right)
\end{gathered}
$$

(other components $=0$ ), where:

$$
\begin{aligned}
& I=3 K_{\mathrm{M}} \phi\left(G_{\mathrm{M}}-G_{\mathrm{C}}\right) \\
& I I=G_{\mathrm{M}}\left(3 K_{\mathrm{M}}+4 G_{\mathrm{C}}\right) \\
& I I I=3 K_{\mathrm{F}} \phi\left(G_{\mathrm{C}}-G_{\mathrm{M}}\right)-G_{\mathrm{C}}\left(3 K_{\mathrm{F}}+4 G_{\mathrm{M}}\right) \\
& I V=G_{\mathrm{M}}\left(3 K_{\mathrm{F}}+4 G_{\mathrm{C}}\right) \\
& D=\left(3 K_{\mathrm{F}}+4 G_{\mathrm{M}}\right)\left(3 K_{\mathrm{M}}+4 G_{\mathrm{C}}\right)- \\
& \quad-12 \phi\left(G_{\mathrm{C}}-G_{\mathrm{M}}\right)\left(K_{\mathrm{F}}-K_{\mathrm{M}}\right) \\
& \varepsilon_{\mathrm{ij}}^{\mathrm{F}}=\left(C T E_{\mathrm{F}}-C T E_{\mathrm{C}}\right) \Delta T \delta_{\mathrm{ij}} \\
& \varepsilon_{\mathrm{ij}}^{\mathrm{M}}=\left(C T E_{\mathrm{M}}-C T E_{\mathrm{C}}\right) \Delta T \delta_{\mathrm{ij}}
\end{aligned}
$$

$K$ being the bulk modulus and $G$ the shear modulus. The subscripts $M, F$ and $C$ refer to the matrix, particle and effective medium, respectively. In our case, we have assumed that the properties of the effective medium are those of the composite. In this work, it was considered that the temperature change $\Delta T$ was $100 \mathrm{~K}$.

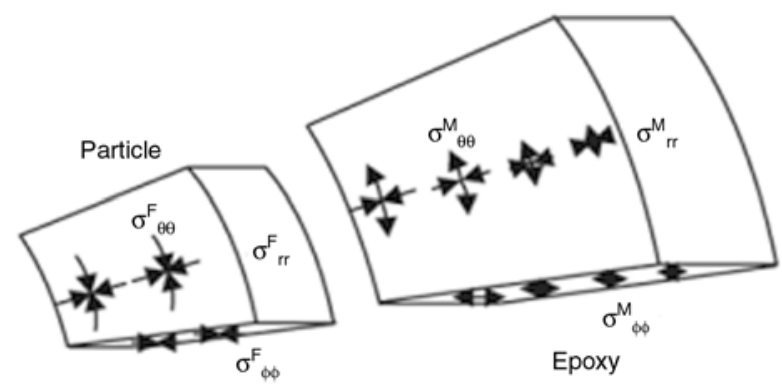

Figure 2. Schematic description of the stress fields acting on the filler and the matrix in a particulate composite

Specifically, for aluminum microparticles as used in the present work $K_{\mathrm{F}}>K_{\mathrm{M}}, G_{\mathrm{F}}>G_{\mathrm{M}}$ and $C T E_{\mathrm{M}}>$ $C T E_{\mathrm{F}}$, and, then, a particle is subjected to a compression radial stress $\sigma_{\text {rr }}^{\mathrm{F}}$ from the epoxy matrix. On the other hand, the shell of the matrix suffers two kinds of stresses: one representing the radial compression of the effective medium; and another corresponding to the radial tensile caused by the particle. A schematic description of the nature of each stress fields acting on the particle (aluminum inclusions in this work) and in the epoxy matrix is presented in Figure 2. During the cooling process from the cure temperature of the composite, compressive stresses operate on the particle. This kind of stress is homogeneous for all particles. Conversely, the strength of stresses present in the matrix diminishes when the distance to the particle increases.

It is known that the chemical shrinkage a polymer suffers during the cure process can also produce residual stresses; however, this contribution is usually neglected since at this stage of the cure process the elastic properties of the matrix are poor. So, to analyze and discuss the data in the present work we only have taken into account the contribution to the stresses coming from the thermal properties of the phases forming the different composites.

Equations (7) and (8) were parameterized in terms of the distance to the center of the particle $r$. In this way, those radial and the tangential stress components which are independent of $r$ (terms $I$ and $I I I$ in the equations above mentioned) have the same sign. Conversely, those terms depending on $r$ (terms $I I$ and $I V$ ) have an opposite sign. It must also be noted that the terms independent of $r$ take into account the influence of the effective medium; i.e., the remainder inclusions into the composite.

On the other hand, when considering that $\phi$ goes to zero and $G_{\mathrm{C}}$ and $C T E_{\mathrm{C}}$ tend to $G_{\mathrm{M}}$ and $C T E_{\mathrm{M}}$ respec- 


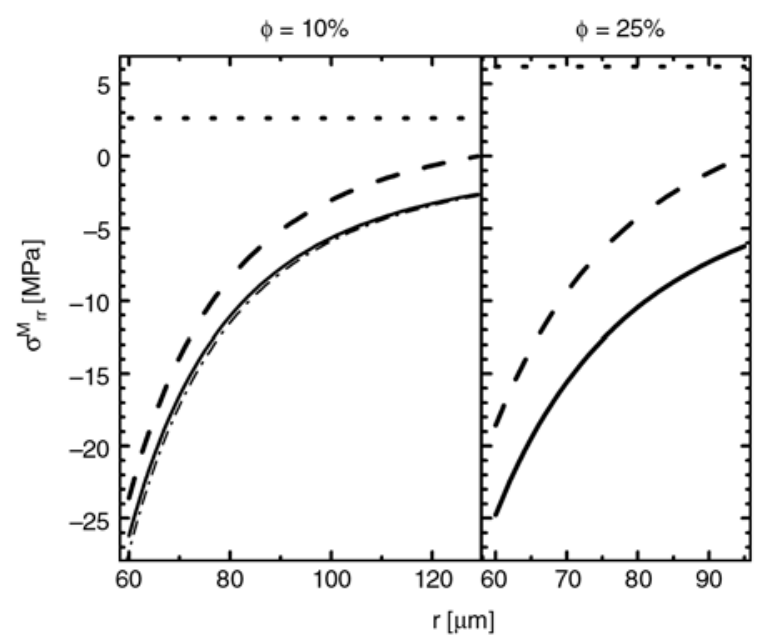

Figure 3. Component $\sigma_{\mathrm{rr}}^{\mathrm{M}}$, represented by $(--)$, of the stress tensor into the matrix as a function of distance to the particle and for different filler contents. In the different plots, the contributions of the terms depending, or not, on $r$ are presented separately, e.g.; the term of $\sigma_{\mathrm{rr}}^{\mathrm{M}}$ that depends on $r$ was represented by (-) and that independent of $r$ by $(\cdot \cdot)$. In the case of $\phi=10 \%$, the stress calculated using the Selsing's equation is also presented by $(-\cdot-)$.

tively, the $\sigma_{\mathrm{rr}}^{\mathrm{M}}$ reduces to the Selsing's equation above discussed.

In Figure 3, the solid line represents the evolution of $\sigma_{\mathrm{rr}}^{\mathrm{M}}$ as a function of $r$ and for different filler contents $\phi$. The particle radius selected was of $60 \mu \mathrm{m}$, chosen taking into account the typical sizes of the aluminum particles used in our composites. In the figure, the different terms contributing to the stress tensor are also plotted. It must be pointed out that for the lower filler content ( $\phi=10 \%)$, the Selsing's equation is also presented. As can be seen in the figure, there is a very good agreement with the tensor term dependent on $r$.

In a second step, on the basis of the results presented above, to calculate the average volumetric radial stresses acting on the matrix as well as the particle we have followed the ideas of Park and Earmme [6]. However, in the present work we focused our analysis on the average volumetric radial stress into the matrix $\left(<\sigma_{\mathrm{Ir}}^{\mathrm{M}}\right)$ where the matrix is characterized by a volume $\left(V^{\mathrm{M}}\right)$. As mentioned, $\phi=\left(r_{\mathrm{f}} / r_{\mathrm{b}}\right)^{3}$; besides the volume of the matrix can be calculated as $V^{\mathrm{M}}=4 / 3 \pi\left(r_{\mathrm{b}}{ }^{3}-r_{\mathrm{f}}{ }^{3}\right)$, then according to Equation (9):

$\left\langle\sigma_{\mathrm{rr}}^{\mathrm{M}}\right\rangle=\frac{1}{V^{\mathrm{M}}} \int \sigma_{\mathrm{rr}}^{\mathrm{M}} \mathrm{d} V$

and using Equation (7) Equation (10) is obtained:

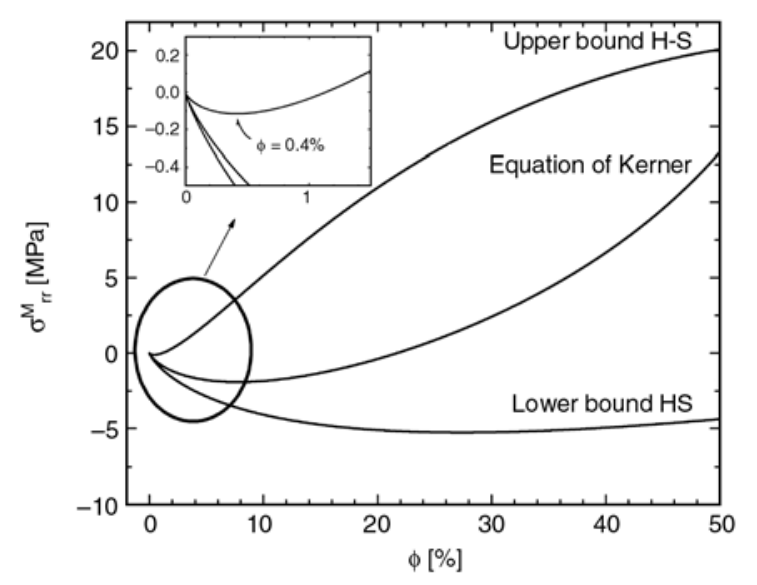

Figure 4. Average volumetric radial stress $\sigma_{\mathrm{rr}}^{\mathrm{M}}$ as a function of the filler content. To this aim, the upper and lower bounds of the Hashin and Shtrikman equation were calculated (see text). Other approximation given by the Kerner equation was also plotted (see section 3.4).

$$
\begin{aligned}
\left\langle\sigma_{\mathrm{rr}}^{\mathrm{M}}\right\rangle= & \frac{12}{D}\left(K_{\mathrm{F}} \varepsilon_{\mathrm{ij}}^{\mathrm{F}} I+K_{\mathrm{M}} \varepsilon^{\mathrm{M}} I I I\right)+ \\
& +\frac{36}{D}\left(-K_{\mathrm{F}} \varepsilon_{\mathrm{ij}}^{\mathrm{F}} I I+K_{\mathrm{M}} \varepsilon_{\mathrm{ij}}^{\mathrm{M}} I V\right) \frac{1}{\frac{1}{\phi}-1}\left(-\ln ^{3} \sqrt{\phi} .\right)
\end{aligned}
$$

The constants $C T E_{\mathrm{C}}, K_{\mathrm{C}}$ and $G_{\mathrm{C}}$ in the effective medium were assumed to be those corresponding to the composite. To calculate these constants, a model developed for particulate composites by Hashin [21] was used. This model allows calculating the bulk $(K)$ and the shear modulus $(G)$ of a particulate composite. Specifically, the model predicts for the different particle content an upper and a lower value (both named bounds) for $K$ and $G$. In Figure 4, the two bounds of $<\sigma_{\mathrm{rr}}^{\mathrm{M}}$ as a function of the filler content were plotted. As can be observed, the lower bound of $\left\langle\sigma_{\mathrm{rr}}^{\mathrm{M}}\right\rangle$ decreases when the filler content increases. After $\phi \approx 25 \%$; $<\sigma_{\mathrm{rr}}^{\mathrm{M}}>$ shows a slight increase with the increment of $\phi$. In the case of the Hashin and Shtrikman (H-S) upper bound and around a filler content of $0.4 \%$, a small minimum in the average volumetric radial stress can be seen; then $<\sigma_{\mathrm{rr}}^{\mathrm{M}}$ rapidly increases when $\phi$ increases.

\subsection{Young's modulus variation due to filler content}

The models discussed in the previous Sections did not give the detailed information we needed to obtain reliable results on the thermal stresses operating in the particulate epoxy composites we are dealing 
Table 1. Young's modulus as a function of filler content of aluminum particles ([22])

\begin{tabular}{|c|c|}
\hline$\phi[\%]$ & Modulus $\mathbf{E}_{\mathbf{C}}[\mathbf{G P a}]$ \\
\hline 5 & $4.3( \pm 0.2)$ \\
\hline 10 & $4.9( \pm 0.2)$ \\
\hline 15 & $5.8( \pm 0.2)$ \\
\hline 20 & $6.2( \pm 0.3)$ \\
\hline 25 & $7.1( \pm 0.2)$ \\
\hline
\end{tabular}

with. To this aim, we have used experimental values of the resonance frequencies measured by using the impulse excitation technique (IET) that we recently reported in [22]. From the mentioned frequencies, the different Young's moduli $(E)$ were calculated. Since it is of utmost importance to understand the different approximations discussed in the present work, we summarize here some of the main results reported in [22]. It is worth remembering that in the case of pure epoxy, a value of $E_{\mathrm{M}}=3.9 \pm 0.2 \mathrm{GPa}$ was found. The corresponding results for the composites are reported in Table 1 . As can be seen, $E_{\mathrm{C}}$ increases when the filler content $(\phi)$ increases.

To fit the experimental data of the Young's modulus, the Kerner equation, generalized by Lewis and Nielsen and Landel [1], was used. It should be noted that the Kerner's equation results from the ratio between the Young's moduli of the composite $\left(E_{\mathrm{C}}\right)$ and the matrix $\left(E_{\mathrm{M}}\right)$, respectively. It is given by Equation (11):

$\frac{E_{\mathrm{C}}}{E_{\mathrm{M}}}=\frac{1+A B \phi}{1-B \psi \phi}$

where $A$ is a constant that takes into account factors as the geometry of the particles and the Poisson's ratio of matrix, $B$ is a constant depending on the relative $E_{\mathrm{F}}$ and $E_{\mathrm{M}}$ values of the filler and matrix phases, respectively. This parameter is defined by Equation (12):

$B=\frac{\frac{E_{\mathrm{F}}}{E_{\mathrm{M}}}-1}{\frac{E_{\mathrm{F}}}{E_{\mathrm{M}}}+A}$

Besides, the factor $\psi$ depends on the maximum packing fraction $\phi_{\mathrm{m}}$ of the filler. $\psi$ can be obtained by using an empirical function as given by Equation (13):

$\psi=1+\frac{1-\phi_{\mathrm{m}}}{\phi_{\mathrm{m}}^{2}} \phi$ where the factor $\phi_{\mathrm{m}}$ was taken from the literature $\phi_{\mathrm{m}}=0.637$ ([22]). This parameter is independent of the materials forming the composite.

The experimental data of $E_{\mathrm{C}}$ as a function of the filler content were fitted using Equation (11) and the constant $A=1.72$ was obtained from the best fit of $E$ versus $\phi$ curve.

Successively, the volumetric radial stress into the matrix given was calculated by means of Equation (10) and using the experimental data obtained by IET. Besides, Equation (14) describing the relationship between the bulk $(K)$ and $E_{\mathrm{C}}$ moduli was used [1]:

$E_{\mathrm{C}}=3 K_{\mathrm{C}}(1-2 \mu)$

where $\mu$ the Poisson's ratio. There is an analogous expression for the shear modulus $G$.

On the other hand, the $C T E_{\mathrm{C}}$ value was calculated using the Hashin-Shtrikman model for each composite.

The evolution of $<\sigma_{\mathrm{rr}}^{\mathrm{M}}$ obtained as a function of $\phi$ is depicted in Figure 4. The estimation of the average volumetric radial stress using the Kerner's equation is also shown. As can be seen, there qualitatively exists a good agreement of the resulting curve with that calculated using the lower Hashin-Shtrikman bound; but, from the comparison the curve corresponding to the Kerner's equation shows a sharper minimum located about a filler content of $8 \%$.

The results presented in the previous sections on the models used to analyze the composites will be used as an input to the analysis of the free nanohole volumes behavior as a function of the filler content into the epoxy matrix composites studied in the present work.

\subsection{Average free nanohole volume variation due to filler content}

The epoxy matrix composites are subjected to a thermal treatment during curing process. Then, if the composite is considered in thermal equilibrium at the high temperature stage, thermal stresses are developed when the samples are cooled to ambient temperature due to the different thermomechanical properties between the matrix and the inclusion. Under this scenario, it is reasonable to expect that the residual stresses have a strong influence onto the average free nanohole volume. Precisely, this assumption allowed Goyanes et al. [8] to satisfacto- 
rily explain the decrease in the $V_{\mathrm{H}}$ versus $\phi$ for aluminum powder contents up to about $15 \%$. However, an explanation regarding highest filler contents is still an open issue.

In order to go deeper into the analysis of the experimental data reported in Figure 1, as a first attempt we assumed a spherical geometry for the aluminum particles. In the above sections, it was found that there is a compressive radial stress around an inclusion.

On the other hand, it has been reported that in polymers the o-Ps lifetime [23] is dominated by the smaller length of the nanohole. Then, in our composites it could be expected that $\tau_{\mathrm{o}-\mathrm{Ps}}$ decreases as a consequence of a diminution of the radial characteristic length of the nanoholes around the particles used as filler.

In a previous paper, authors of the present work [8] used a simple relationship between the free volume fraction and the average hydrostatic pressure $P$, given by Equation (15):

$f=f^{0}+\beta \cdot P$

where $f^{0}$ is the free volume fraction into the pressurefree state and $\beta$ is the free volume compressibility.

The free volume fraction and the average volume of the nanoholes are related by $f=N_{\mathrm{h}} \cdot V_{\mathrm{H}}$ where $N_{\mathrm{h}}$ is the number of nanoholes per volume unit. If $N_{\mathrm{h}}$ does not change with the filler content, then the change of $V_{\mathrm{H}}$ is proportional to the pressure with a constant of the order of $\beta / N_{\mathrm{h}}$. However, there has not been reported a relation between the average radial stress in the matrix $\left\langle\sigma_{\mathrm{rr}}^{\mathrm{M}}>\right.$ and $V_{\mathrm{H}}$. In such a way, assuming the linear relation between $f$ and $P$ we propose the following relationship (Equation (16)) that allows calculating the free nanohole volume as a function of the average volumetric radial stress:

$$
V_{\mathrm{H}}=V_{\mathrm{H}}^{0}+C\left\langle\sigma_{\mathrm{rr}}^{\mathrm{M}}\right\rangle
$$

where $C$ is a constant and $V_{\mathrm{H}}^{0}$ the free nanohole volume in the stress free state. As already mentioned, the different $V_{\mathrm{H}}$ can be directly obtained from the $\tau_{\mathrm{o}-\mathrm{Ps}}$ experimental values.

Taking into account Equation (16), $V_{\mathrm{H}}$ and $<\sigma_{\mathrm{rr}}^{\mathrm{M}}>$ should have the same dependence of the filler content $\phi$. Then, the experimental data of Figure 1 were fitted in the $\phi$ range from 0 to about $15 \%$ using

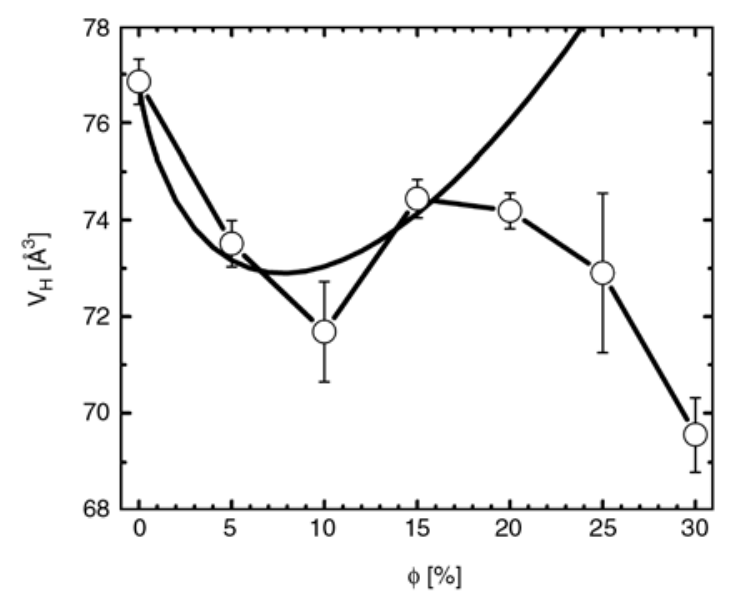

Figure 5. Dependence of the average free nanohole volume as a function of the filler content: $(-\mathrm{O}-)$ experimental values and $(-)$ calculated data obtained using Equation (16)

Equation (16) with $C$ as a free parameter. The $<\sigma_{\mathrm{rr}}^{\mathrm{M}}$ values were calculated by means of Equation (10). From the fit, the parameter $C$ results $2.06 \cdot 10^{-6} \AA^{3} \cdot \mathrm{Pa}^{-1}$. In Figure 5, the mentioned function is presented with a solid line. As can be seen, qualitatively there exists a reasonable agreement between the fitting curve and that representing the experimental data in the $\phi$ range from 0 to about $15 \%$. For higher $\phi$ values, the fitting function overestimates the experimental $V_{\mathrm{H}}$ values. In fact, for $\phi>15 \%$ the average free nanohole volume predicted shows a strong increase while the experimental data decrease.

Summarizing, it is clear that the model used is not good enough to satisfactorily fit the whole behavior of the $V_{\mathrm{H}}$ experimental data as a function of the filler content. As a consequence, we considered necessary to introduce other elements in order to explain the whole behavior of the $V_{\mathrm{H}}$ versus $\phi$ curve. Therefore, in the next section other contributions to the average free nanohole volume, especially those that take into account the inclusion-matrix interphases are analyzed.

\subsection{Characterization of the inclusion-matrix interphases using microscopy techniques}

In this Section, information regarding the inclusionmatrix interphases present in the composites is analyzed.

\subsubsection{A scanning electron microscopy study}

The composites studied were microstructurally characterized using Scanning Electron Microscopy (SEM). In Figure 6, as an example some SEM 


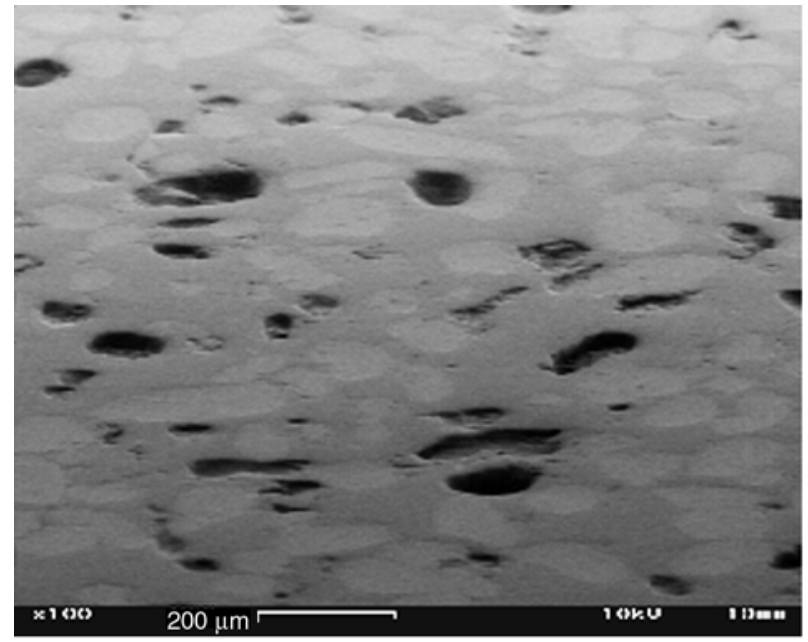

a)

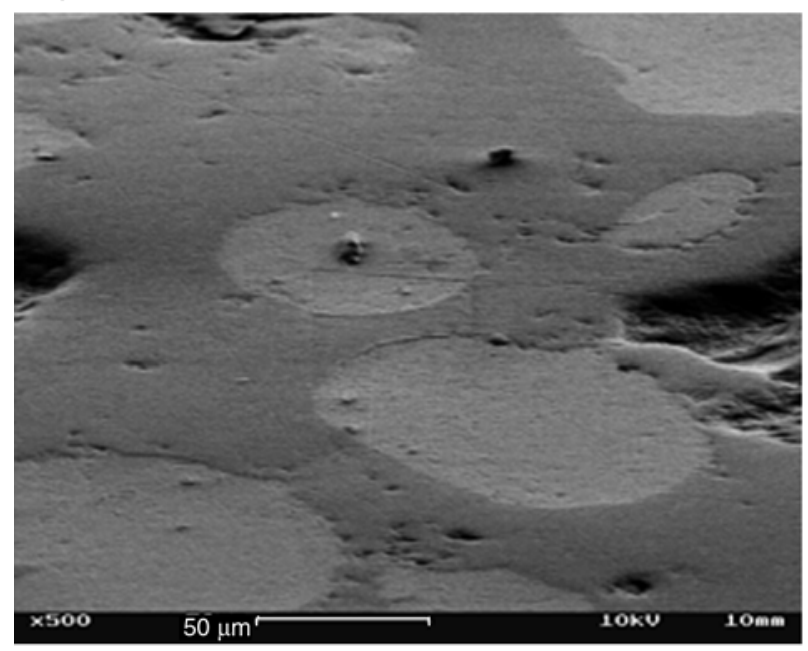

c)

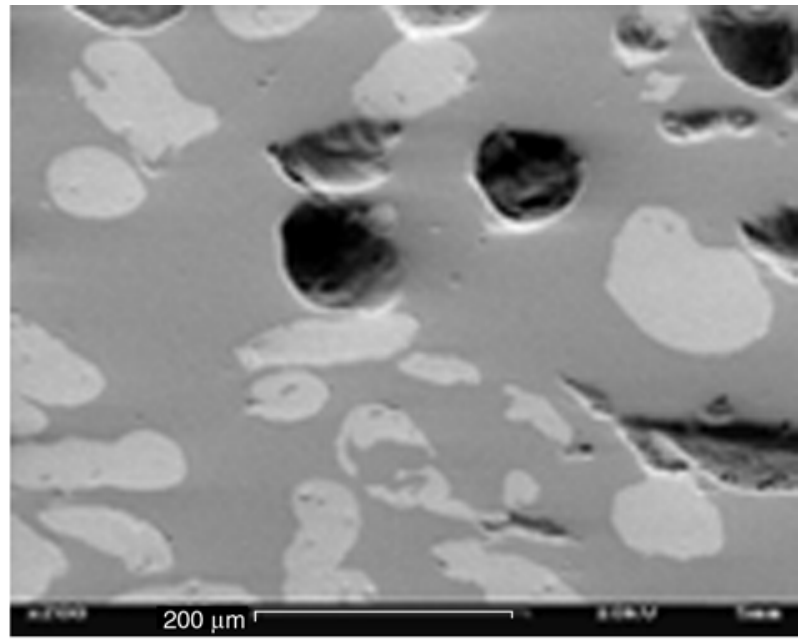

b)

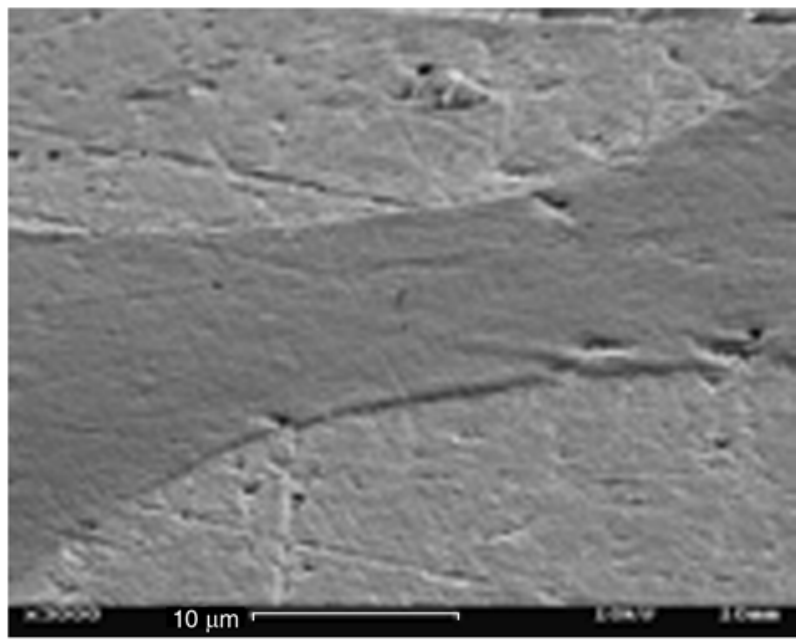

d)

Figure 6. SEM micrographs of an epoxy composite (30\% aluminum particle content). The images were obtained using different magnifications: 100, 200, 500 and 3000.

micrographs obtained for a sample containing 30\% of aluminum particles are shown. In these images, the surface of aluminum particles is clearer than the matrix one. Dark holes correspond to particles removed from the matrix during the surface polishing. In the images, it can be observed that the matrix presents a surface smoother than that of the inclusion. This is a consequence that the matrix is softer than the particle. Besides, it seems that the particles are slightly deeper than the level of the matrix. To explore in detail this behavior, atomic force microscopy was used. The results are presented in the next section.

\subsubsection{An atomic force microscopy study}

Atomic force microscopy was used to study the topography of the composites. The use of this technique allowed us to go further into the analysis of differences between the surface levels of the parti- cles with respect to the matrix. In this Section, we present AFM results obtained for the samples filled with $30 \%$ of particles. In Figure 7, images of the topography and shift phase, taken along the dashed line drawn in the images, obtained for the samples mentioned above are shown.

Analyzing the topographic mapping, it results that the bright zones in the images represent a surface level higher than that of the dark zones. As can be observed, in both samples the inclusion presents an image darker than that corresponding to the matrix surrounding the filler. Therefore, it can be concluded that the inclusions are located at a deeper level than that of the matrix. This behavior is in total agreement with the SEM results presented in the previous Section.

On the other hand, in the figure the profile of height $Z$ as a function of the $x$ length parameter, considered along the dashed line drawn in the image, show that 

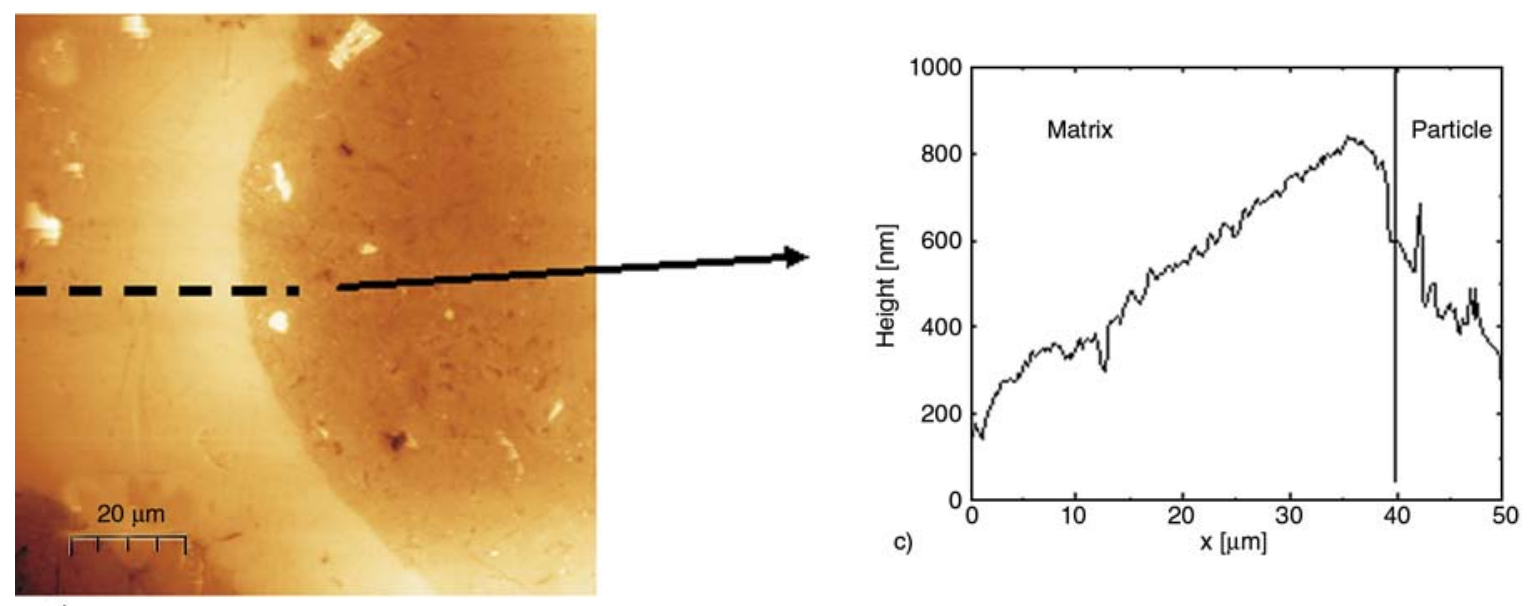

a)
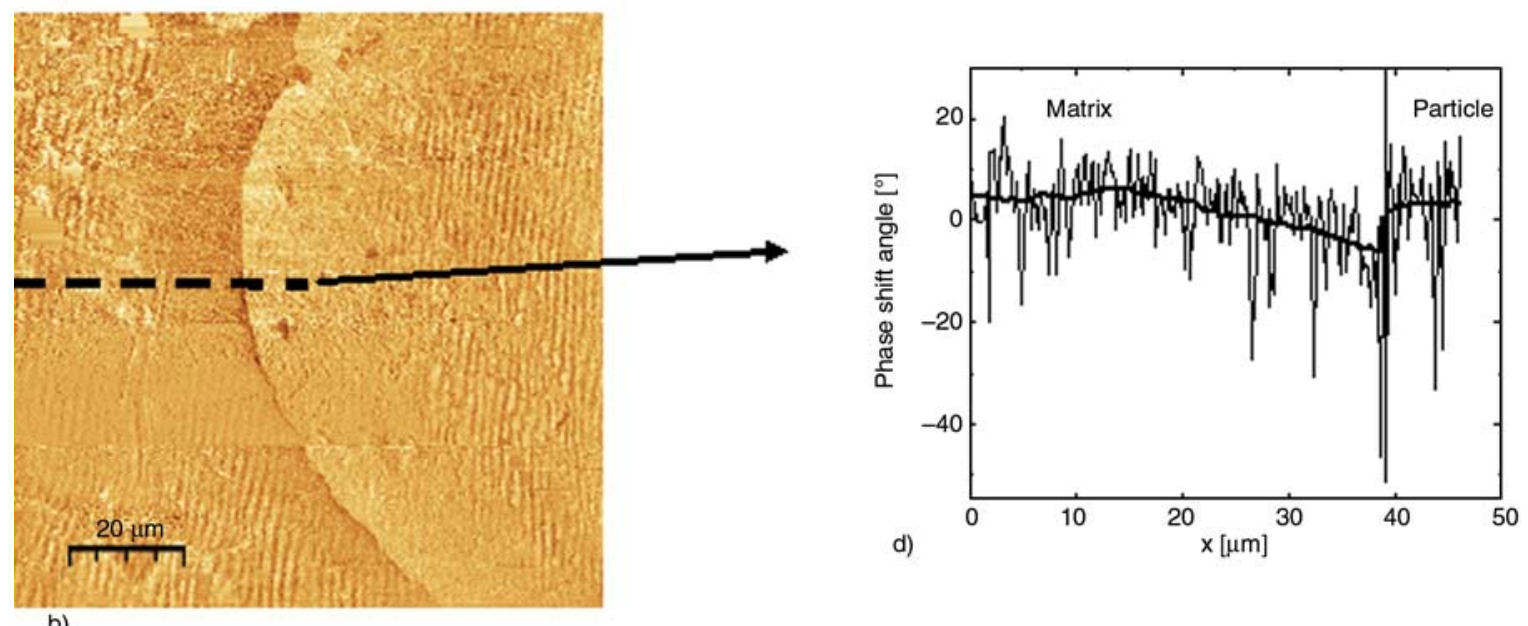

b)

Figure 7. AFM measurements of an epoxy composite (30\% filler content). a) topographic mapping. b) phase shift mapping around an aluminum particle. In c) and d) the profile of height $Z$ and the phase shift, along the axis $x$ represented by the dashed lines drawn in the images, are respectively shown. The solid line in d) was obtained smoothing the experimental data.

the maximum values of $Z$ are reached around the inclusion. When moving outside the particle towards the matrix, a systematic decrease of $Z$ is observed. Into the particle, $Z$ rapidly decreases in about 500 $600 \mathrm{~nm}$. This behavior seems abnormal and must be carefully analyzed. In fact, as the inclusion is harder than the matrix, it would be expected that this last one would be located in a deeper level than that corresponding to the particle. We consider that such a difference could be explained in terms of the relaxation induced in the matrix by the cutting procedure. Specifically, when a composite sample is cut the stresses around a particle are relaxed. Therefore, a relaxation of the tangential stresses around a particle would cause an 'expansion' of the matrix in the direction normal to the surface, i.e., a relative change in the level of the surfaces corresponding to the inclusion and the matrix. This analysis is compatible with the scheme of the stresses operating in a composite represented in Figure 2, and with the contraction of the radial dimension of the nanohole. On the other hand, the phase image (Figure 7b) clearly shows that matrix and particle have different phase angles. Although the profile (phase angle versus $x$ ) obtained from the respective images are very noisy, after smooth experimental data when moving outside the particle towards the matrix (up to $20 \mu \mathrm{m}$ ) a small but significant variation of the phase shift around the inclusion is observed.

It is known that the phase shifts depend on the energy dissipated in the tapping interaction of probe and the specimen explored [24]. Differences in the phase shifts are usually associated with differences in surface or in underlying properties (stiffness, viscosity and adhesion). Hence, the phase shift is sensitive to variations in materials local properties and it can be an important tool for surface analysis [25]. 


\subsection{Evidence on the inclusion-matrix interphases from DSC measurements}

In order to completely explain the behavior of $V_{\mathrm{H}}$ as a function of the filler content plotted in Figure 1, we have taken into account an additional element from which some information had been obtained using AFM: the inclusion-matrix interphase. On the basis of previous results, it was assumed that around the filler particles there exists a region with different properties from those of the matrix. This interphase region was characterized using differential scanning calorimetry (DSC). Specifically and following ideas reported in the literature, the interphase volumetric fraction was estimated from the DSC curves [10, 26, 27].

To simplify our study, the geometry of the particles used as filler of the epoxy-based composites was assumed to be spherical with a characteristic radius $r_{f}$. It was also supposed that each particle was surrounded by an interphase with an associated thickness $\Delta r_{\mathrm{i}}$. On the basis of the experimental techniques used to study the characteristics of the interphases, we have not considered chemical factors like the presence of oxides onto the inclusion surfaces. Work in progress is addressed to the study of the chemical and physical characteristics that should be considered into the inclusion-matrix interphases contribution.

In a previous work, we reported the different $\Delta r_{\mathrm{i}}$ values as well the volumetric fraction of the interphases $v_{\mathrm{i}}$ as a function of the filler content for the same composites studied here [13]. Additionally, in the mentioned paper we already obtained the glass transition temperatures $T_{\mathrm{g}}$ for each composite sample. Experimentally, DSC data were obtained using a heating rate of $10 \mathrm{~K} / \mathrm{min}$, in a temperature range between 293 and $453 \mathrm{~K}$. Specifically, the glass transition temperature was determined of the onset temperature for the step observed in the thermograms.

Table 2. Thickness, volumetric fraction of interphase and glass transition temperature for different filler content of particles of aluminum in the epoxy-resin composite ([13])

\begin{tabular}{|c|c|c|c|}
\hline $\boldsymbol{\phi}[\mathbf{\%}]$ & $\Delta \mathbf{r}_{\mathbf{i}}[\boldsymbol{\mu m}]$ & $\mathbf{v}_{\mathbf{i}}^{\mathbf{D S C}}[\mathbf{\%}]$ & $\mathbf{T}_{\mathbf{g}}[\mathbf{K}]$ \\
\hline 0 & 0 & 0 & $391( \pm 1)$ \\
\hline 5 & $0.2( \pm 0.1)$ & $0.05( \pm 0.03)$ & $392( \pm 1)$ \\
\hline 10 & $0.3( \pm 0.1)$ & $0.16( \pm 0.05)$ & $392( \pm 2)$ \\
\hline 15 & $0.4( \pm 0.1)$ & $0.30( \pm 0.08)$ & $393( \pm 1)$ \\
\hline 20 & $2.18( \pm 0.08)$ & $2.26( \pm 0.08)$ & $393( \pm 1)$ \\
\hline 25 & $2.9( \pm 0.6)$ & $3.8( \pm 0.8)$ & $393( \pm 1)$ \\
\hline 30 & $6.3( \pm 0.7)$ & $10( \pm 1)$ & $395( \pm 2)$ \\
\hline
\end{tabular}

In Table 2, the results of the parameters above mentioned are presented.

As useful for the general discussion on the whole data reported in this work, we briefly analyze the data reported in Table 2. As can be seen, $\Delta r_{i}$ and its associated volume $v_{\mathrm{i}}$ increase when the filler content increases. For $\phi \leq 15 \%$ both parameters show a small increment but for higher filler contents they strongly increase. It is important to point out that the highest value of $\Delta r_{\mathrm{i}}(\sim 6 \mu \mathrm{m})$ approximately represents $10 \%$ of a typical radius of an inclusion. Furthermore, the maximum value of $v_{\mathrm{i}}$ is equivalent to $15 \% \mathrm{ca}$. of the total volume of the epoxy matrix. In addition, the glass transition temperature systematically increases with the increment of $\phi$.

From the DSC results shown in Table 2, we can conclude that the effect of the interphase becomes significant for filler contents higher than $15 \%$. At this point, it is worth remembering that in this $\phi$ region the $V_{\mathrm{H}}$ versus $\phi$ behavior cannot be described using the thermal stress model. Therefore, an additional term in Equation (16) should be considered; i.e., a contribution to $V_{\mathrm{H}}$ related to the inclusionmatrix interphase. Recently, from PALS measurements on the same samples studied in the present work we reported quantitative information on this term [13]. Specifically, studying a sample containing $15 \%$ of filler, a bimodal-like distribution of the free nanohole volume was obtained. So, nanohole volume values of $76 \AA^{3}$ (representing the free volume in the epoxy resin) and $32 \AA^{3}$ (assigned to the free volume in the interphase region) were reported.

\subsection{Influence of the inclusion-matrix interphases on the average free nanohole volumes}

In this Section, the different results previously obtained on the aluminum inclusion-epoxy resin matrix interphases are used in order to analyze the behavior of the average free nanohole volume in the whole range of the filler content used to charge the matrix of the different composites. As already shown, the free nanohole volume associated with the interphase is lower than that of the bulk matrix. Therefore, the average free nanohole volume of a composite $V_{\mathrm{H}}$ (e.g., that corresponding to the matrix) should diminish with the increase of the interphase contribution as a consequence of a progressive increase of the filler content. 
To evaluate the interphase influence on $V_{\mathrm{H}}$, as a first approximation a homogeneous number of holes distributed into the matrix of the composite, independently of the position of the inclusion, was considered. Then, we have modeled the average free nanohole volume as follows: a contribution due to the average of free nanohole volume of the matrix outward the interphase (i.e. bulk) $V_{\mathrm{H} \text { bulk }}$ and another coming from the average free nanohole volume corresponding to the interphase $V_{\mathrm{H} \text { Int }}$.

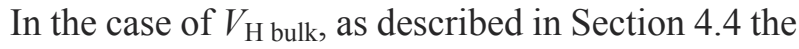
value of this parameter was estimated using the average radial stress. On the other hand, as a first approximation $V_{\mathrm{H} \text { Int }}$ was considered to be constant and its value was that coming from the analysis of bimodal distribution of the PALS data (specifically $32 \AA^{3}$ ). Then, the average free nanohole volume was calculated as a combination of the two free nanohole volume terms above mentioned according to Equation (17):

$$
V_{\mathrm{H}}=\frac{V_{\mathrm{HInt}} \cdot v_{\mathrm{i}}+V_{\mathrm{H} \text { bulk }} \cdot v_{\text {bulk }}}{v_{\mathrm{i}}+v_{\text {bulk }}}
$$

In this equation, $v_{\mathrm{i}}$ is the volume fraction associated with the inclusion-matrix interphases and $v_{\text {bulk }}$ volume fraction of the bulk. To link both parameters, we have used the following relationship $v_{\text {bulk }}=$ $1-\phi-v_{\mathrm{i}}$, which is a reasonable one.

The volume fraction values associated with the inclusion-matrix interphases were obtained from the DSC data reported in Table 2. But, to use them into the general model developed to fit the $V_{\mathrm{H}}$ versus $\phi$ data presented in Figure 1, a scale constant factor which allowed matching the data was used. This factor was obtained from a free parameter fitting.

Finally, using the model simply expressed by Equation (17) the experimental values of $V_{\mathrm{H}}$ obtained from PALS measurement as a function of the filler content in the different composites studied could be satisfactorily described in the whole volume range of aluminum particles contained in the different composites (see Figure 8). Undoubtedly, for $\phi>15 \%$ the contribution of the inclusion-matrix interphases becomes very important but, as we have discussed in detail along the present work, $\phi \leq 15 \%$ the average free nanohole volume was mainly due to the contribution of the radial thermal stresses.

Though we have analyzed our data under the assumption that all metallic particles used as filler

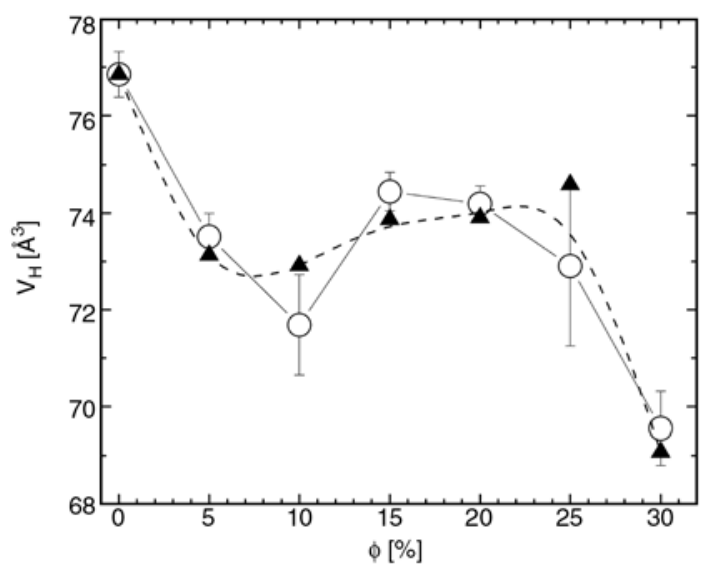

Figure 8. Dependence of average free nanohole volume as a function of the filler content: (-०—) experimental values obtained from PALS; (- $\mathbf{\Delta}-)$ data obtained from Equation (17) in which the contribution of the average thermal stresses and that of the inclusion-matrix interphases were considered. The dashed line is an eye guide

of the epoxy matrix were spherical and had the same size, the results predicted by the model used allowed us to satisfactory fit the experimental behavior of the free nanohole volume as a function of the filler content. Work in progress will allow us to evaluate the influence of the real geometry of the particles and their distribution of sizes on the free nanohole volume.

\section{Conclusions}

In the present work and on the basis of previous studies on the particulate epoxy matrix composites recently published $[13,22]$, we have made a systematic study on the evolution of the free nanohole volumes in particulate epoxy matrix composites with the filler content ranging from 0 to $30 \%$ of volumetric fraction. Specifically, as filler we have used aluminum particles with characteristic sizes of about $100 \mu \mathrm{m}$.

As a starting point of the present work, experimental free nanohole volume data directly measured using positron annihilation lifetime spectroscopy recently reported, were used. In a second step, these data were interpreted in the frame of different wellknown micromechanical models.

As a first approximation, when considering only the contribution of the thermal stresses acting into the composite as a consequence of the fabrication process and the further curing treatments applied to the resulting composites, we could only satisfactory explain the evolution of the free nanohole volume 
in the range of filler content between $0 \%$ and approximately $15 \%$. For highest filler contents, the approach used failed. For this reason, from the different micromechanical models available in the literature that allow to calculate the thermal stresses in composites, in the present work we choose the Park-Earmme model which we specifically adapted to take into account the contribution of the different stresses coming from the phases present in the epoxy composite.

To go further, in some cases it was necessary to use experimental data as input parameters into the model used. While values of the Young's modulus used here were taken from our recently published paper on the same composites studied in this work [22]. There is no available quantitative information on matrix-particle interphases. Therefore, we obtained specific experimental information using atomic force and scanning electronic microscopies, differential scanning calorimetry and a refined analysis of the positron annihilation lifetime data. It is worth mentioning that in the present work the concept of interphase was used in a general context; thus, chemical and physical properties of the interphases did not are separately evaluated.

Among the information reported in the present work on particulate metal-epoxy matrix composites, a contribution to the knowledge of these materials that deserves special consideration is the study about the influence of the matrix-particle interactions on the nanohole sizes in epoxy-based composites.

Summarizing, the present work allowed to completely describe the behavior of the free nanohole volume as a function of the inclusion content into the composites. In fact, when considering the joint contribution of the radial stresses in epoxy matrix and the particle-matrix interphase, the reformulated micromechanical Park and Earmme model allowed us to give a satisfactory description of the free nanohole volume behavior in the whole range of the filler content.

\section{Acknowledgements}

This work was partially supported by Agencia Nacional de Promoción Científica y Tecnológica (PICT 2011-1088), Comisión de Investigaciones Científicas de la Provincia de Buenos Aires, Universidad Nacional del Centro de la Provincia de Buenos Aires (Argentina) and Universidad de Buenos Aires (Argentina) (Research Project 2006-2009 X808).

\section{References}

[1] Nielsen L. E., Landel R. F: Mechanical properties of polymers and composites. Marcel Dekker, New York (1994).

[2] Harsch M., Karger-Kocsis J., Holst M.: Influence of fillers and additives on the cure kinetics of an epoxy/ anhydride resin. European Polymer Journal, 43, 11681178 (2007).

DOI: $10.1016 /$ j.eurpolymj.2007.01.025

[3] Eshelby J. D.: The determination of the elastic field of an ellipsoidal inclusion, and related problems. Proceedings of Royal Society of London A, 241, 376-396 (1957).

DOI: $10.1098 /$ rspa.1957.0133

[4] Selsing J.: Internal stresses in ceramics. Journal of the American Ceramic Society, 44, 419 (1961) DOI: 10.1111/j.1151-2916.1961.tb15475.x

[5] Christensen R. M., Lo K. H.: Solutions for effective shear properties in three phase sphere and cylinder models. Journal of the Mechanics and Physics of Solids, 27, 315-330 (1979).

DOI: 10.1016/0022-5096(79)90032-2

[6] Park S. J., Earmme Y. Y.: Bound solution for residual stress due to thermal mismatch in particle reinforced composites. Journal of Composite Materials, 33, 12051221 (1999).

DOI: $10.1177 / 002199839903301303$

[7] Low I. M.: Toughening of epoxies by thermal expansion mismatch. Journal of Applied Polymer Science, 39, 759-762 (1990).

DOI: 10.1002/app.1990.070390324

[8] Goyanes S., Rubiolo G., Salgueiro W., Somoza A.: On the free volume evolution in a deformed epoxy composite. A positron annihilation study. Polymer, 46, 9081-9087 (2005).

DOI: $10.1016 /$ j.polymer.2005.07.020

[9] Gao S-L., Mäder E.: Characterisation of interphase nanoscale property variations in glass fibre reinforced polypropylene and epoxy resin composites. Composites Part A: Applied Science and Manufacturing, 33, 559-576 (2002).

DOI: $10.1016 / \mathrm{S} 1359-835 \mathrm{X}(01) 00134-8$

[10] Theocaris P. S.: The unfolding model for the representation of the mesophase layer in composites. Journal of Applied Polymer Science, 30, 621-645 (1985). DOI: $10.1002 /$ app.1985.070300214

[11] Pérez-Pacheco E., Moreno-Chulim M. V., ValadezGonzález A., Rios-Soberanis C. R., Herrera-Franco P. J.: Effect of the interphase microstructure on the behavior of carbon fiber/epoxy resin model composite in a thermal environment. Journal of Materials Science, 46, 4026-4033 (2011).

DOI: $10.1007 / \mathrm{s} 10853-011-5331-0$ 
[12] Goyanes S., Rubiolo G., Marzocca A., Salgueiro W., Somoza A., Consolati G., Mondragon I.: Yield and internal stresses in aluminum filled epoxy resin. A compression test and positron annihilation analysis. Polymer, 44, 3193-3199 (2003).

DOI: $10.1016 /$ S0032-3861(03)00229-5

[13] Tognana S., Salgueiro W., Somoza A.: On the matrixparticle interphase in epoxy-based composites. Journal of Alloys and Compounds, 495, 588-591 (2010).

DOI: $10.1016 /$ j.jallcom.2009.10.058

[14] Jean Y.: Characterizing free volumes and holes in polymers by positron annihilation spectroscopy. in 'Positron spectroscopy of solids' (eds.: Dupasquier A., Mills Jr A. P.) IOS Press, Amsterdam, 563-580 (1995).

[15] Mallon P. E.: Applications to polymers. in 'Principles and application of positron and positronium chemistry' (eds.: Jean Y. C., Mallon P. E., Schrader D. M.) World Scientific, Singapore, 253-280 (2003).

[16] Ferry J. D.: Viscoelastic properties of polymers. John Wiley and Sons, New York (1980).

[17] Dlubek G., Stejny J., Alam M. A.: Effect of cross-linking on the free-volume properties of diethylene glycol bis(allyl carbonate) polymer networks: A positron annihilation lifetime study. Macromolecules, 31, 4574 4580 (1998).

DOI: $10.1021 / \mathrm{ma} 971801 \mathrm{y}$

[18] Salgueiro W., Marzocca A., Somoza A., Consolati G., Cerveny S., Quasso F., Goyanes S.: Dependence of the network structure of cured styrene butadiene rubber on the sulphur content. Polymer, 45, 6037-6044 (2004). DOI: $10.1016 /$ j.polymer.2004.05.008

[19] Horcas I., Fernández R., Gómez-Rodríguez J. M., Colchero J., Gómez-Herrero J., Baro A. M.: WSXM: A software for scanning probe microscopy and a tool for nanotechnology. Review of Scientific Instruments, 78, 013705/1-013705/8 (2007).

DOI: $\underline{10.1063 / 1.2432410}$
[20] Mastelaro V. R., Zanotto E. D.: Residual stresses in a soda-lime-silica glass-ceramic. Journal of Non-Crystalline Solids, 194, 297-304 (1996).

DOI: 10.1016/0022-3093(95)00509-9

[21] Hashin Z:: Analysis of composite materials - A survey. Journal of Applied Mechanics, 50, 481-505 (1983).

DOI: 10.1115/1.3167081

[22] Tognana S., Salgueiro W., Somoza A., Marzocca A.: Measurement of the Young's modulus in particulate epoxy composites using the impulse excitation technique. Materials Science and Engineering: A, 527, 4619-4623 (2010).

DOI: $10.1016 /$ j.msea.2010.04.083

[23] Soles C. L., Yee A. F.: A discussion of the molecular mechanisms of moisture transport in epoxy resins. Journal Polymer Science Part B: Polymer Physics, 38, 792-802 (2000).

DOI: 10.1002/(SICI)1099-0488(20000301)38:5<792::

$$
\text { AID-POLB16>3.0.CO;2-H }
$$

[24] Cleveland J. P., Anczykowski B., Schmid A. E., Elings V. B.: Energy dissipation in tapping-mode atomic force microscopy. Applied Physics Letters, 72, 26132615 (1998).

DOI: $10.1063 / 1.121434$

[25] Babcock K. L., Prater C. B.: Phase imaging: Beyond topography. Veeco, Santa Barbara (1995).

[26] Theocaris P. S., Stavroulakis G. E., Panagiotopoulos P. D.: Calculation of effective transverse elastic moduli of fiber-reinforced composites by numerical homogenization. Composites Science and Technology, 57, 573-586 (1997).

DOI: $10.1016 / \mathrm{S} 0266-3538(97) 00018-3$

[27] Lombardo N.: Effect of an inhomogeneous interphase on the thermal expansion coefficient of a particulate composite. Composites Science and Technology, 65 , 2118-2128 (2005).

DOI: $\underline{10.1016 / \mathrm{j} . c o m p s c i t e c h .2005 .05 .006}$ 